\title{
Multiphase imaging of gas flow in a nanoporous material using
}

\section{remote detection NMR}

\author{
Elad Harel, Josef Granwehr, Juliette A. Seeley, and Alex Pines ${ }^{1}$ \\ Materials Sciences Division, Lawrence Berkeley National Laboratory, and Department of Chemistry, \\ University of California, Berkeley, California 94720 \\ 1e-mail: pines@cchem.berkeley.edu
}

Pore structure and connectivity determine how microstructured materials perform in applications such as catalysis, fluid storage and transport, filtering, or as reactors. We report a model study on silica aerogel using a recently introduced time-of-flight (TOF) magnetic resonance imaging technique to characterize the flow field and elucidate the effects of heterogeneities in the pore structure on gas flow and dispersion with ${ }^{129} \mathrm{Xe}$ as the gas-phase sensor. The observed chemical shift allows the separate visualization of unrestricted xenon and xenon confined in the pores of the aerogel. The asymmetrical nature of the dispersion pattern alludes to the existence of a stationary and a flow regime in the aerogel. An exchange time constant is determined to characterize the gas transfer between them. As a general methodology, this technique provides new insights into the dynamics of flow in porous media where multiple phases or chemical species may be present. 
Understanding multiphase fluid transport in porous materials is necessary to gain insight into a wide range of physical processes from drug delivery and distribution in blood vessels ${ }^{1}$, fluid mixing in chemical reactors ${ }^{2}$, to fuel and exhaust transport in fuel cells $^{3}$. General methods for mapping the dynamics of multicomponent flow are rare, primarily due to the difficulty of probing past the surface layer and in distinguishing different chemical species ${ }^{4}$. Studies of flow in porous materials, therefore, are often misleading because they generally only provide bulk structural information, like pore volumes and pore sizes, or kinetic and thermodynamic properties, while it is the microscopic structure that determines transport properties ${ }^{5}$.

Silica aerogel is a low-density material with a high porosity and a large internal surface area that has been studied extensively owing to its unique structural, elastic, optical, and thermal properties ${ }^{6,7}$. Many applications for aerogels have been proposed ranging from biocompatible scaffolds as biosensors ${ }^{8}$, aerosol ${ }^{9}$ and chemical ${ }^{10}$ detectors to catalytic substrates ${ }^{11}$ and gas adsorption media ${ }^{12}$. Its preparation involves sol-gel hydrolysis followed by condensation, solvent exchange, and supercritical drying ${ }^{13}$. Densities typically range from 0.003 to $0.25 \mathrm{~g} / \mathrm{cm}^{3}$, while specific surface areas can be as large as $1000 \mathrm{~m}^{2} / \mathrm{g} .{ }^{14}$ Aerogel is built of small, microporous $(<2 \mathrm{~nm})$ particles that aggregate to form a large fractal network ${ }^{15,16}$. This network is mesoporous $(2-50 \mathrm{~nm})$ and builds clusters, which themselves aggregate ${ }^{17}$ to constitute macropores (>50 nm).

Different techniques are used to characterize the pore structure of aerogels. Neutron and X-ray scattering methods ${ }^{15,16}$ provide information about various structural features like mean particle and pore sizes and their distribution, network morphology, or the determination of the skeletal density. But the pore connectivity, which is important 
for fluid flow, is not directly accessible. Transmission electron microscopy (TEM) ${ }^{18}$ and atomic force microscopy (AFM) ${ }^{17}$ provide a detailed picture of the pore space on the surface, but only examine a very limited region of the sample. Adsorption, permeability, and dynamic gas expansion experiments ${ }^{19}$ are common methods to obtain average pore dimensions and pore connectivity. These techniques provide reliable relative pore dimensions when comparing different samples, but the results systematically vary from scattering data. Despite extensive research over the last two decades in this field, determining how the microscopic structure relates to its unique chemical and physical properties has proven a tremendous challenge ${ }^{20}$. One reason seems to be the difficulties in distinguishing between mesopores and macropores with the established analytical methods.

The majority of spectroscopic methods are of limited use for the study of aerogels since they require optical access past the surface layer. Nuclear magnetic resonance (NMR) and magnetic resonance imaging (MRI), on the other hand, are especially suited to study opaque or otherwise optically inaccessible materials ${ }^{21,22}$, as long as radio frequency (RF) radiation can penetrate the material and provided that spin-active nuclei are present. The high porosity of aerogel allows the use of a tracer compound, such as ${ }^{129} \mathrm{Xe}$ gas, to act as a sensor of its structure and chemistry ${ }^{23,24}$. Several studies have attempted to link the chemical shift exhibited by ${ }^{129} \mathrm{Xe}$ in contact with aerogel with its structural characteristics ${ }^{5,25}$. In some cases multiple peaks were observed with widely varying linewidths ${ }^{26}$, depending on the exact nature of the sample. While for micropores the chemical shift is expected to reflect the void space available to the xenon atom ${ }^{27}$, the smaller chemical shift values observed in meso- and macropores depend on the pore 
geometry, the sorption energy, and the temperature ${ }^{25,28}$. But as a general rule, for a given material a larger chemical shift always corresponds to a smaller pore $\operatorname{size}^{29}$.

The sensitivity of NMR experiments of porous samples can be improved by physically separating the encoding and detection steps, which allows the individual optimization of each ${ }^{30}$. With remote detection, information about a stationary sample is encoded onto the spin magnetization of a flowing NMR-active sensor medium ${ }^{31,32}$. This magnetization is then read out with an optimized detector as the sensor medium flows out of the sample. Remote detection is particularly useful for fluid flow studies ${ }^{33}$, where spectroscopic or imaging information about a porous environment can be correlated with the time-of-flight (TOF) of the fluid from its encoding location to the detector ${ }^{34}$. This gives a spatially or spectrally resolved dispersion pattern, effectively mapping the flow field. For each encoding step, the dispersion can be resolved stroboscopically by applying multiple detection cycles as the fluid flows through the detector (Fig. 1).

In this work, we apply remote detection MRI with chemical shift selective encoding to distinguish between xenon gas occluded in the aerogel's silicate matrix and free gas that flows without impediment. Differences in the dispersion patterns of xenon inside and outside the aerogel are used to model the flow, and the local gas flow velocity allows for estimation of the effective porosity of the aerogel. When taking into account known structural properties of the aerogel, the flow features can be correlated with the structure of the material. Our technique makes this possible despite the fact that the spatial resolution of the image encoding is several orders of magnitude too low to resolve the aerogel's pore structure. 


\section{RESULTS AND DISCUSSION}

Figure 2A shows an NMR spectrum recorded remotely with continuously flowing gas, and a 2D representation of the spectral versus the dispersion dimensions. A distinct aerogel peak is apparent approximately $40 \mathrm{ppm}$ downfield from the free gas peak, corresponding to a mesoporous average pore size in the range of $20-30 \mathrm{~nm} \cdot{ }^{29}$ In the center of the figure, a schematic of the aerogel sample and sample housing is shown. The flow direction is parallel to the static magnetic field pointed along $z$.

In Fig. 2B the chemical-shift selective projections onto a plane perpendicular to the lateral $y$ dimension are displayed, showing the time-averaged image projections of free and occluded xenon gas. By applying a selective RF pulse at the appropriate frequency only spins from a select region of the spectrum are excited. In this way, free and occluded gas are separately visualized. The 2D data sets in Fig. 2C and D show the dispersion of the free and occluded gas along the flow dimension, respectively. The width of the shapes indicates the extent of dispersion, while the slope is a measure of the flow velocity. The longitudinal flow velocity of the free gas at the inlet, $v_{\mathrm{i}}=2.5 \pm 0.6 \mathrm{~cm} / \mathrm{s}$, is about a factor of four slower than that at the outlet, $v_{0}=10.4 \pm 0.6 \mathrm{~cm} / \mathrm{s}$, consistent with the principle of mass balance as the diameter of the tube at the inlet is a factor of two wider than at the outlet. The shapes are consistent with unrestricted flow with the inlet showing more dispersion than the outlet since it has a longer flow path, and the gas from the inlet has to pass the tube-narrowing constriction at the outlet. The occluded gas exhibits a qualitatively different behavior: it remains inside the sample for a time longer than it takes most of the free gas from the inlet to reach the detection region. 
Full three-dimensional isosurfaces of the data are shown in Fig. 3A and Supplementary Information Video 1. Each image corresponds to the origin of the gas that arrives at the detector a certain time after encoding. With increased TOF, the gas originating a greater distance from the detector is visualized. Figure 3B shows snapshots of free gas using a slice-selective phase encoding pulse sequence ${ }^{34}$ with an increased spatial and temporal resolution. Alongside each image is a projection lateral to the flow direction. From the first image at $400 \mathrm{~ms}$ only spins located at the outlet side of the sample are visible. For longer TOF, gas from the inlet side gradually reaches the detector. No significant signal intensity from the free gas is observed inside the center of the aerogel. A rough estimate revealed the average flow velocity of gas in the aerogel to be a factor of seven faster than expected with unrestricted flow in void space of the same volume. The rapid flow velocity is indicative of a channeling mechanism through the aerogel sample through a well-connected pore network.

Figure 4A shows TOF dispersion curves of free gas from voxels at the inlet and the outlet of the aerogel, and Fig. 4B shows the same data of occluded gas from a voxel inside the aerogel. Without flow resistance, convection dominates the free gas flow, and dispersion is mainly due to laminar flow inside the cylindrically shaped tubing on either side of the aerogel sample. Dispersion is commonly modeled by a propagator that describes the displacement of a fluid within a given time. The four-dimensional data available in our experiments, in principle, contains dispersion information in the spatial as well as temporal domain without any conversion. Dispersion in the spatial domain in an unrestricted geometry can be modeled by a normal distribution ${ }^{35}$, using a Gaussian propagator. In the time domain an analytic description reveals that a normal distribution 
is only an approximate model, which becomes less valid at very slow flow velocities or very short flow distances. Experimentally it was found that the dispersion curve at the outlet can be well modeled with a normal distribution, $s(t, \boldsymbol{r})=s_{0}(\boldsymbol{r}) \Delta t \exp \left(-\left(t-t_{0}(\boldsymbol{r})\right)^{2} / 2 \sigma(\boldsymbol{r})^{2}\right) / \sqrt{2 \pi} \sigma(\boldsymbol{r})$, where $s_{0}(\boldsymbol{r})$ is the signal amplitude, $\Delta t$ is the time between detection pulses, $\sigma(\boldsymbol{r})^{2}$ is the variance of the distribution at the spatial coordinate $\boldsymbol{r}$ of the encoded spins, $t$ is the time-of-flight between encoding and detection, and $t_{0}$ is the center of the dispersion pattern. Both $t_{0}$ and $\sigma$ depend on $\boldsymbol{r}$ since they are functions of the flow path. Because of the constrictions in the flow path, it is not straightforward to interpret $\sigma$ or correlate it with the molecular diffusion coefficient $D_{0}$. However, $t_{0}$ and the knowledge of $\boldsymbol{r}$ in our experiments can be used to calculate the mean longitudinal flow velocity as $v(z)=\Delta z / \Delta t_{0}$, where $\Delta z=z_{n}-Z_{n-1}$ is the distance between adjacent data points, $n$ and $n-1$, along the $z$ dimension, and $\Delta t_{0}=t_{0}\left(z_{n}\right)-t_{0}\left(z_{n-1}\right)$.

The dispersion of the free gas at the inlet can also be modeled reasonably well with a normal distribution. The dispersion of the gas originating within the aerogel, however, deviates greatly from a normal distribution, and its width is broader than the width of the free gas from the inlet of the aerogel whose flow path is necessarily longer. Since in a homogeneous medium the dispersion must increase with time and distance traveled, a decreasing dispersion width with increasing flow distance is an indication of the existence of two different flow paths with different dispersion mechanisms, path lengths, or flow velocities. Examination of the rising edge of the dispersion pattern in Fig. 4B reveals that it has a slope comparable to the free gas, but its tail is significantly longer. This indicates that a major fraction of the gas flows through the aerogel without significant resistance, while the peak at $40 \mathrm{ppm}$ primarily originates from gas that is 
lingering inside the aerogel for some time. Once released into the pores constituting the flow field, these xenon atoms have a low probability of being occluded again, and rapidly flow by convection through the sample. This accounts for the asymmetric shape of the peak at $40 \mathrm{ppm}$.

To model the dispersion pattern of the occluded gas, let us assume that after the encoding step, which we consider for simplicity as happening instantaneously, xenon atoms remain occluded for a time $T$ before being released to the flow field. The distribution of $T$ shall be given by a correlation time, $\tau(\boldsymbol{r})$, which describes the time constant for the release of occluded gas, so that the dispersion-free signal is given by $s_{0}{ }^{\prime}(\boldsymbol{r}, T)=s_{0}(\boldsymbol{r}) \Delta t \exp (-T / \tau(\boldsymbol{r})) / \tau(\boldsymbol{r})$. The observed signal can then be calculated by convoluting $s_{0}{ }^{\prime}(r, T)$ with the dispersion propagator of the free gas from the same location as

$$
\begin{aligned}
s(t, \boldsymbol{r}) & =\frac{s_{0}}{\sqrt{2 \pi} \tau(\boldsymbol{r}) \sigma(\boldsymbol{r})} \int_{0}^{\infty} \exp \left(-\frac{T}{\tau(\boldsymbol{r})}\right) \exp \left(-\frac{\left(t-t_{0}(\boldsymbol{r})-T\right)^{2}}{2 \sigma(\boldsymbol{r})^{2}}\right) d T \\
& =\frac{s_{0}}{2 \tau(\boldsymbol{r})} \exp \left(\frac{\sigma(\boldsymbol{r})^{2}}{2 \tau(\boldsymbol{r})^{2}}-\frac{t-t_{0}(\boldsymbol{r})}{\tau(\boldsymbol{r})}\right)\left[1-\operatorname{erf}\left(\frac{\sigma(\boldsymbol{r})}{\sqrt{2} \tau(\boldsymbol{r})}-\frac{t-t_{0}(\boldsymbol{r})}{\sqrt{2} \sigma(\boldsymbol{r})}\right)\right]
\end{aligned}
$$

Using this expression, the dispersion curves of occluded gas can be modeled fairly well (Fig. 4B). Note that while the occluded gas can be observed directly, the existence of a separate flow field domain can only be probed indirectly with the current data. While $\tau(\boldsymbol{r})$ quantifies the connectivity between two different pore domains within the sample, $t_{0}(\boldsymbol{r})$ and $\sigma(\boldsymbol{r})$ can be used to characterize the nature of the main flow field. Fitting the aerogel data with Eq. (1) allows us to indirectly obtain $\sigma(\boldsymbol{r})$ and $t_{0}(\boldsymbol{r})$ of the flow field inside the aerogel. Because $\sigma(\boldsymbol{r})$ and $t_{0}(\boldsymbol{r})$ are both parameters that characterize gas flow of free gas, we could expect them to be at least somewhat correlated, while the presence or absence 
of correlation between $t_{0}(\boldsymbol{r})$ and $\tau(\boldsymbol{r})$ might allow conclusions about structural correlations between the different pore domains. The knowledge of the spatial dependence of $t_{0}$ enables an estimate of the local longitudinal flow velocity $v_{\mathrm{a}}$ inside the aerogel and also how well a certain volume element in the aerogel is connected to the flow field. By comparing this velocity with the xenon velocity at the outlet of the sample, the effective porosity $\phi_{\mathrm{e}}$, i.e. the volume fraction of the pore space that is fully interconnected and contributes to the fluid flow through the aerogel, can be calculated as $\phi_{\mathrm{e}}=r v_{\mathrm{o}} / \nu_{\mathrm{a}}$, where $r \approx 0.25$ is the ratio of the cross-sections of the outlet tube and the aerogel. Experimentally, $v_{\mathrm{a}}=23.5 \pm 3 \mathrm{~cm} / \mathrm{s}$ was obtained as an average value for the whole sample, giving $\phi_{\mathrm{e}} \approx 0.11 \pm 0.02$. Comparing $\sigma(\boldsymbol{r})$ of occluded and free gas at locations where both are visible reveals similar values of the two. This further supports the model that occluded gas diffuses out of the mesopores into the flow field.

We can use Eq. (1) to fit each voxel inside the aerogel individually. Figure 4C shows $\tau$ in different slices perpendicular to the gas flow direction. A uniform value of $\tau$ is not obtained throughout the sample volume. As a general trend, $\tau$ along $z$ is largest in the center, where it shows a large distribution of different values in the plane perpendicular to $z$, while it decreases towards the inlet and outlet, where it shows much more uniform values. Inside the sample large heterogeneities can be observed that are seemingly uncorrelated with the distance from the sample wall. It is likely that heterogeneities in the pore connectivity of the sample are responsible for these latter effects. Structural defects such as cracks or fissures are less likely since these would manifest themselves as additional signal in the free gas images and at the same time regions of depleted signal in the occluded gas images, which is not observed. 
Fitting $t_{0}$ over the whole sample reveals major heterogeneities as well, shown in Fig. 4D. Note that the variation of $t_{0}$ within a single slice can be remarkably large, indicating local variations of the flow velocity. This could be caused by a varying permeability of the pore network that constitutes the flow field, suggesting the existence of pores with a large local distribution of diameters. However, as the flow field could be characterized only indirectly, further investigation is warranted.

From the estimated value of $\phi_{\mathrm{e}}$, one would expect a significant signal from xenon in the flow field. Since the signal of the free gas vanishes almost completely inside the aerogel, it seems possible that ${ }^{129} \mathrm{Xe}$ in pores constituting the flow field covers a similar spectral region as xenon that is stationary in the aerogel, but maybe causes a broader line. This would be consistent with a structural model that distinguishes between mesopores and macropores. In light of this data, it may be useful to distinguish these two pore domains not purely by their diameter, but also by their connectivity.

As can be seen by comparing Figs. 4C and 4D, there is no obvious correlation between $t_{0}$ and $\tau$, which indicates that heterogeneities of the pore domains are not necessarily related. In Fig. 4F, this lack of correlation is visualized by plotting $\tau$ vs. $t_{0}$ of the different data points. In contrast, plotting $\sigma$ vs. $t_{0}$ (Fig. 4E) shows a clear correlation between the two. But this correlation is relatively noisy, which could be explained if structural heterogeneities responsible for the variation of $t_{0}$ and $\sigma$ were on a smaller length scale than the resolution of the image, as a distribution of different $t_{0}$ tends to broaden $\sigma$. Data in [23] indicates that this is a realistic option. 


\section{CONCLUSION AND OUTLOOK}

Time-of-flight NMR studies with remote detection, using the chemical shift as a contrast property, was shown to be a powerful tool for studying fluid flow inside nanoporous materials. Chemical selectivity was achieved either by using selective pulses to address only nuclei with a certain chemical shift, or by resolving the full spectrum of the sample as an additional experimental dimension.

The dispersion of xenon gas encoded inside the aerogel allowed us to distinguish two different flow components, one governed by convection and the other one by diffusion. While the majority of the gas that passes the sample is driven by convection at an elevated velocity due to a reduced effective porosity of the pores constituting the flow field, the larger fraction of gas that is inside the sample at any time is in the diffusive regime with a much longer residence time. The gas exchange between the two domains is slow, explaining the large values of $\tau$. This could be explained by the existence of closed pores that are poorly interconnected to a network of open pores. This would also explain the discrepancy between gas expansion experiments, which commonly use a capillary model to interpret the data, and scattering experiments in predicting average pore dimensions ${ }^{19}$. It seems plausible to identify the closed pores as mesopores and the open pores as macropores, but we do not have direct evidence for this assignment yet.

The transient detection of dispersion allows local time constants to be measured, which can be used to obtain spatially resolved values of what are typically considered bulk material properties. The addition of chemical selectivity makes this a general approach for studying fluid dynamics in three dimensions inside porous materials of multiple-phase or multi-component mixtures. 


\section{MATERIALS}

\section{CHEMICALS}

The aerogel sample had a density of $0.008-0.009 \mathrm{~g} / \mathrm{cm}^{3}$ and measured roughly $0.5 \mathrm{~cm}^{3}$ in volume. A gas mixture of $1 \% \mathrm{Xe}$ (natural abundance), $10 \% \mathrm{~N}_{2}$, and $89 \% \mathrm{He}$ is passed through a cell containing optically pumped rubidium vapor.

\section{EXPERIMENTAL SETUP}

All experiments were performed on a Unity Inova spectrometer (Varian Inc., Palo Alto, CA) at a magnetic field of 7.05 $\mathrm{T}$, corresponding to a ${ }^{129} \mathrm{Xe}$ frequency of $82.9 \mathrm{MHz}$. The homebuilt detection probe was placed from atop the magnet, while the microimaging probe (Varian Inc., Palo Alto, CA) was placed inside a gradient stack (Resonance Research Inc., Billerica, MA) from below. The upper rim of the encoding and the lower rim of the detection coils were separated by approximately $2 \mathrm{~cm}$. The detection coil was kept inside an isolating copper shield to minimize mutual couplings with the encoding coil. The sample was housed in a Teflon casing and is surrounded by clay to keep it stationary and to prevent flow around the sample. The outlet of the sample housing was $6.3 \mathrm{~mm}$ while the inlet was $12.7 \mathrm{~mm}$ in diameter. A XenoSpin polarizer (Amersham Health, Durham, NC) optically pumped ${ }^{129}$ Xe to about $10 \%$ polarization. Xenon then flowed through the sample, where it was encoded. After passing the detection coil it was vented to the atmosphere. The pressure and flow rate could be controlled with the use of a mass flow meter and valves ${ }^{36}$. The imaging data was obtained at a pressure of 4.0 bar and 0.65 standard liter-per-minute mass flow rate, while the spectroscopic TOF data was obtained at a pressure of 2.0 bar at the same mass flow rate.

\section{PULSE SEQUENCES}

Two-dimensional spectroscopic data was obtained by applying a $\pi / 2$ excitation pulse followed by a variable time delay before the application of another $\pi / 2$ pulse to prevent the spin magnetization from dephasing as it flows to the detector. As the Xenon arrives at the detection region, its magnetization is measured time-resolved using a train of $\pi / 2$ pulses separated by $50 \mathrm{~ms}$. Three-dimensional imaging data and their respective projection were obtained by phase encoding along $x, y$, and $z$ using field gradient pulses of $(200 \mu$ s) length. Slice selective phase encoding data in Fig. 4B was obtained by phase encoding along $x$ and $z$, while selecting a $0.5 \mathrm{~cm}$ slice of spins along $y$ with a sinc pulse in the presence of a y-gradient. For chemical shift selectivity a selective gauss excitation pulse with a bandwidth of $2 \mathrm{kHz}$ was used. A four step phase cycle was used to obtain frequency discrimination in the indirect dimensions and to 
remove the baseline caused by unencoded $\operatorname{gas}^{37}$. A full three-dimensional experiment took less than one hour, while a two-dimensional spectroscopic experiment could be performed in about eight minutes.

\section{Acknowledgments}

We would like to thank S. Garcia for help with probe hardware, and P.N. Sen and V.V. Telkki for helpful discussions.

E. H. is supported by a fellowship from the U.S. Department of Homeland Security under DOE contract number DEAC05-00OR22750. This work is supported by the Director, Office of Science, Office of Basic Energy Sciences, Materials Sciences and Nuclear Sciences Divisions of the U.S. Department of Energy under contract DE-AC0376 SF0098.

\section{Competing financial interests}

The authors declare that they have no competing financial interests. 


\section{REFERENCES}

${ }^{1}$ S.H. Jang, M.G. Wientjes, D. Lu, J.L.-S. Au, "Drug Delivery and Transport to Solid Tumors", Pharmaceutical Research 20, 1337-1350 (2003)

${ }^{2}$ L.F. Gladden, M.H.M. Lim, M.D. Mantle, A.J. Sederman, E.H. Stitt, "MRI visualization of two-phase flow in structured supports and trickle-bed reactors", Catalysis Today 79, 203-210 (2003)

${ }^{3}$ B.C.H. Steele, A. Heinzel, "Materials for fuel-cell technologies", Nature 414, 345-352 (2001)

${ }^{4}$ R.W. Mair, C.-H. Tseng, G.P. Wong, D.G. Cory, R.L. Walsworth, "Magnetic resonance imaging of convection in laser-polarized xenon", Phys. Rev. E, 61, 2741-2748 (2000)

5 D.M. Gregory, R.E. Gerald, R.E. Botto, "Pore-structure determinations of silica aerogels by ${ }^{129}$ Xe NMR spectroscopy and imaging", J. Magn. Reson. 131, 327-335 (1998)

${ }^{6}$ V. Gibiat, O. Lefeuvre, T. Woignier, J. Pelous, J. Phalippou, “Acoustic properties and potential applications of silica aerogels", J. Non-Cryst. Solids, 186, 244-255 (1995)

${ }^{7}$ D.W. Hua, J. Anderson, J. Di Gregorio, D.M. Smith, G. Beaucage, "Structural analysis of silica aerogels", J. Non-Cryst. Solids, 186, 142-148 (1995)

${ }^{8}$ M. Power, B. Hosticka, E. Black, C. Daitch, P. Norris, "Aerogels as biosensors: viral particle detection by bacteria immovilized on large pore aerogel”, J. Non-Cryst. Solids, 285, 303-308 (2001)

${ }^{9}$ M. Guise, B. Hosticka, B. Earp, P.M. Norris, “An Experimental Investigation of Aerosol Collection Utilizing Packed Beds of Silica Aerogel Microspheres." J. Non-Cryst. Solids, 285, 317-322 (2001)

${ }^{10}$ T. Sumiyoshi, I. Adachi, R. Enomoto, T. Iijima, R. Suda, M. Yokoyama, H. Yokogawa, "Silica aerogels in high energy physics", J. Non-Cryst. Solids, 225, 369-374 (1998)

11 J. Fricke, ed., Aerogels, Springer Proceedings in Physics, Vol. 6 (Springer, Berlin, 1986)

12 B. Hosticka, P.M. Norris, J.S. Brenizer, C.E. Daitch, "Gas flow through aerogels”, J. Non-Cryst. Solids, 225, 293-297 (1998)

13 A.J. Hunt, K.D. Lofftus, "Process considerations in monolithic aerogels" (D.E. Clark, D.R. Ulrich, Eds.), Better Ceramics Through Chemistry III, Vol. 121, p. 679, Materials Research Society, Pittsburgh (1998)

${ }^{14}$ H. El Rassy, A.C. Pierre, "NMR and IR spectroscopy of silica aerogels with different hydrophobic characteristics", J. Non-Cryst. Solids, 351, 1603-1610 (2005)

${ }^{15}$ A. Emmerling, J. Fricke, "Small angle scattering and the structure of aerogels", J. Non-Cryst. Solids 145, 113-120 (1992)

${ }^{16}$ D.W. Schaefer, K.D. Keefer, "Structure of Random Porous Materials: Silica Aerogel", Phys. Rev. Lett. 56, 2199-2202 (1986)

${ }^{17}$ C. Marlière, F. Despetis, P. Etienne, T. Woignier, P. Dieudonné, J. Phalippou, "Very large-scale structures in sintered silica aerogels as evidenced by atomic force microscopy and ultra-small angle X-ray scattering experiments" J. Non-Cryst. Solids, 285 148-153 (2001) 
${ }^{18}$ G.C. Ruben, L.W. Hrubesh, T.M. Tillotson, "High-resolution transmission electron microscopy nanostructure of condensed silica-aerogel”, J. Non-Cryst. Solids, 186, 209-218 (1995)

${ }^{19}$ G. Reichenauer, C. Stumpf, J. Fricke, "Characterization of $\mathrm{SiO}_{2}$, RF and carbon aerogels by dynamic gas expansion", J. Non-Cryst. Solids 186, 334-341 (1995)

20 S. Gavalda, K. Kaneko, K.T. Thomson, K.E. Gubbins, "Molecular modeling of carbon aerogels", Colloids and Surfaces A: Physicochemical and Engineering Aspects 187, 531-538 (2001)

21 Callaghan P.T. Principles of Nuclear Magnetic Resonance Microscopy Oxford University Press; 1993

${ }^{22}$ R.W. Mair, G.P. Wong, D. Hoffmann, M.D. Hurlimann, S. Patz, L.M. Schwartz, R.L. Walsworth, "Probing porous media with gas diffusion NMR", Phys. Rev. Lett., 83, 3324-3327

23 L.G. Kaiser, T. Meersmann, J.W. Logan, A. Pines, "Visualization of gas flow and diffusion in porous media”, Proc. Natl. Acad. Sci. USA, 97, 2414-2418 (2000)

${ }^{24}$ G. Guillot, P.-J. Nacher, G. Tastevin, "NMR diffusion of hyperpolarized ${ }^{3} \mathrm{He}$ in aerogel: a systematic pressure study", Magn. Reson. Imag. 19, 391-394 (2001)

${ }^{25}$ V.V. Terskikh, I.L. Mudrakovskii, V.M. Mastikhin, ${ }^{129}$ Xe Nuclear Magnetic Resonance Studies of the Porous Structure of Silica Gels", J. Chem. Soc. Faraday Trans. 89, 4239-4243 (1993)

${ }^{26}$ I.L. Moudrakovski, C.I. Ratcliffe, J.A. Ripmeester, L.-Q. Wang, G.J. Exarhos, T.F. Baumann, J.H. Satcher, "Nuclear Magnetic Resonance Studies of Resorcinol-Formaldehyde Aerogels", J. Phys. Chem. B 109, 11215-11222 (2005)

27 J. Demarquay, J. Fraissard, "Xe-129 NMR of xenon adsorbed on zeolites - relationship between the chemical-shift and the void space”, Chem. Phys. Lett., 136, 314-318 (1987)

${ }^{28}$ J.A. Ripmeester, C.I. Ratcliffe, "On the Application of ${ }^{129}$ Xe NMR to the Study of Microporous Solids", J. Phys. Chem. 94, 7652-7656 (1990)

${ }^{29}$ V.V Terskikh, I.K. Moudrakovski, S.R. Breeze, S. Lang, C. I. Ratcliffe, J. A. Ripmeester, A. Sayari, “A General Correlation for the ${ }^{129}$ Xe NMR Chemical Shift-Pore Size Relationship in Porous Silica-Based Materials", Langmuir, 18 5653-5656 (2002)

${ }^{30}$ D.B. Zax, A. Bielecki, K.W. Zilm, A. Pines, D.P. Weitekamp, “Zero field NMR and NQR”, J. Chem. Phys. 83, 4877 (1985)

31 A. J. Moulé, M. M. Spence, S. Han, J.A. Seeley, K.L. Pierce, S. Saxena, A. Pines, “Amplification of xenon NMR and MRI by remote detection”, Proc. Natl. Acad. Sci. USA 100, 9122-9127 (2003)

32 J.A. Seeley, S. Han, A. Pines, "Remote detected high-field MRI of porous samples", J. Magn. Reson. 167, 282-290 (2004)

33 I.V. Koptyug, S.A. Altobelli, E. Fukushima, A.V. Matveev, R.Z. Sagdeev, "Thermally polarized H-1 NMR microimaging studies of liquid and gas flow in monolithic catalysts", J. Magn. Reson. 147, 36-42 (2000) 
${ }^{34}$ J. Granwehr, E. Harel, S. Han, S. Garcia, A. Pines, P.N. Sen, Y.-Q. Song, "Time-of-flight flow imaging using NMR remote detection", Phys. Rev. Lett. 95, 075503 (2005)

${ }^{35}$ G. Taylor, "Dispersion of soluble matter in solvent flowing slowly through a tube", Proc. Royal Soc. (London) A 219, 186-203 (1953)

${ }^{36}$ S. Han, S. Garcia, T.J. Lowery, E.J. Ruiz, J. Seeley, L. Chavez, D.S. King, D. Wemmer, A. Pines, "Improved NMR based bio-sensing with optimized delivery of polarized 129 Xe to solutions", Anal. Chem. 77, 4008-4012 (2005)

${ }^{37}$ J. Granwehr, J.T. Urban, A.H. Trabesinger, A. Pines, "NMR detection using laser-polarized xenon as a dipolar sensor", J. Magn. Reson. 176, 125-139 (2005) 


\section{FIGURE CAPTIONS}

Figure 1: Basic remote detection pulse sequence. The spins initially point along the static magnetic field, $\mathbf{B}_{0}$, parallel to the flow direction. A. A selective pulse excites the spins of interest. B. After an evolution period $\tau_{\text {delay }}$ a $\pi / 2$ pulse is applied, effectively storing one component of the magnetization $\mathbf{B}_{0}$, preventing its dephasing. C. As the spins travel to the detection region all transverse magnetization is dephased, leaving only longitudinal magnetization. A train of $\pi / 2$ detection pulses is applied at regular intervals to measure the time at which encoded spins arrive at the detection coil. D. The amplitude of each FID provides one point along the dispersion curve, with dips when the encoded gas arrives at the detection region. E. $\tau_{\text {delay }}$ for spectroscopic experiments or the strength of pulsed field gradients (applied between the encoding and the storage pulse, not shown in the figure) for imaging experiments were linearly changed along the indirect dimension. The Fourier transform of the time domain or $k$-space signal provides the spectrum or image, respectively.

Figure 2: A. One-dimensional ${ }^{129}$ Xe NMR spectrum and the corresponding TOF resolved spectrum of xenon flowing through aerogel. In this latter experiment a $\pi / 2$ pulse encoded all the spins, and the interferogram was obtained in the indirect dimension by arraying the delay time between encoding and storage to obtain information about all chemical-shift species. The aerogel, whose outline is plotted in green, was held in place by a Teflon sample holder as shown in the schematic on the right. B. Chemicalshift selective pulses were applied during encoding to select either free xenon or occluded xenon. Both images are averaged along the TOF dimension and along $y$ from the full, four-dimensional data sets. The field-of-view was taken as $3 \mathrm{~cm} \times 3 \mathrm{~cm} \times 5 \mathrm{~cm}$ with a resolution of $5 \mathrm{~mm}$. C. and D. Chemical shift selective pulses were applied to either free xenon (C.) or occluded xenon (D.), and a three-dimensional remote experiment was performed for each. Each image is averaged along the transverse dimensions relative to the direction of flow resulting in the TOF of spins from slices along $z$. White lines through the inlet and outlet region indicate the least-squares regression line, or the mean flow velocity in each region along $z$. The width or spread of each region indicates the extent of dispersion.

Figure 3: A. Three-dimensional, time-resolved isosurfaces of occluded (blue) and free gas (red) up to 1 second after encoding. B. 2-dimensional images from slice-selective experiments showing channeling of free gas through the aerogel. Each image was taken with a spatial resolution of $3 \mathrm{~mm}$ along $x$ and $z$, respectively, and with a time resolution of $50 \mathrm{~ms}$. The outline in blue indicates the location of the aerogel sample as determined from selectively exciting only occluded spins.

Figure 4: A. Dispersion of free xenon encoded at the outlet $(x)$ and inlet $(\Delta)$ of the aerogel. The parameters obtained from fits of a normal distribution to each of the two data sets (black curves) were $t_{0}=0.41 \mathrm{~s}$ and $\sigma=0.08 \mathrm{~s}$ for the outlet data, and $t_{0}=0.53 \mathrm{~s}$ and $\sigma=0.12 \mathrm{~s}$ for the inlet data. B. Dispersion of occluded gas in the center of the sample (o). Signal intensity is present even after the last detection pulse is applied. A fit using Eq. (2) provided $t_{0}=0.41 \mathrm{~s}, \sigma=0.07 \mathrm{~s}$, and $\tau=0.62 \mathrm{~s}$ (magenta). C. $\tau$ for different slices through the aerogel perpendicular to $z$. Red colors correspond to short $\tau$ and blue to long $\tau$, as shown by the colorbar. The gray shade outlines the aerogel sample. D. Analogous representation of $t_{0}$ in different slices perpendicular to z. E. Plot of $\sigma$ vs. $t_{0}$. A linear regression gives a correlation coefficient of 0.7 . F. Plot of $\tau$ vs. $t_{0}$. No significant correlation could be identified between the two. 


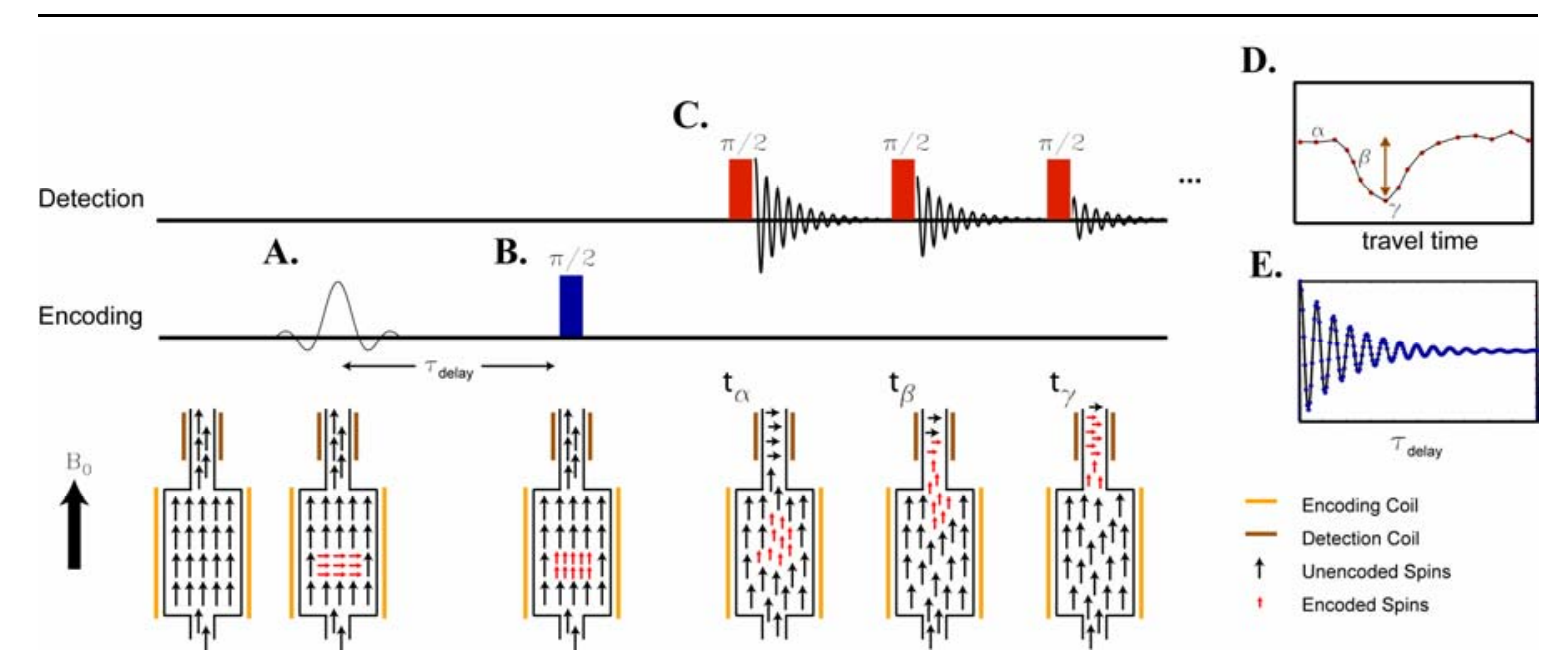

FIGURE 1 

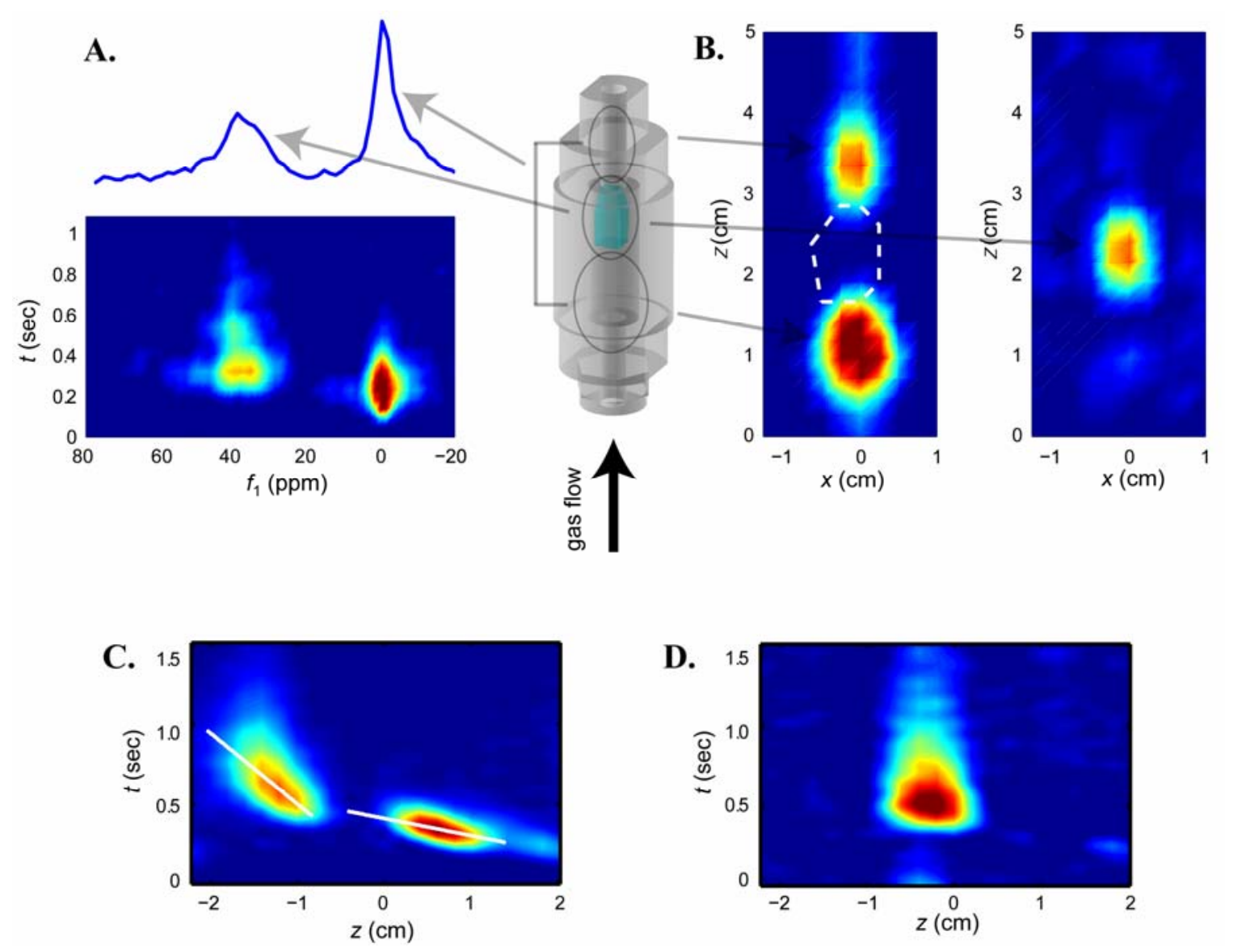

FIGURE 2 


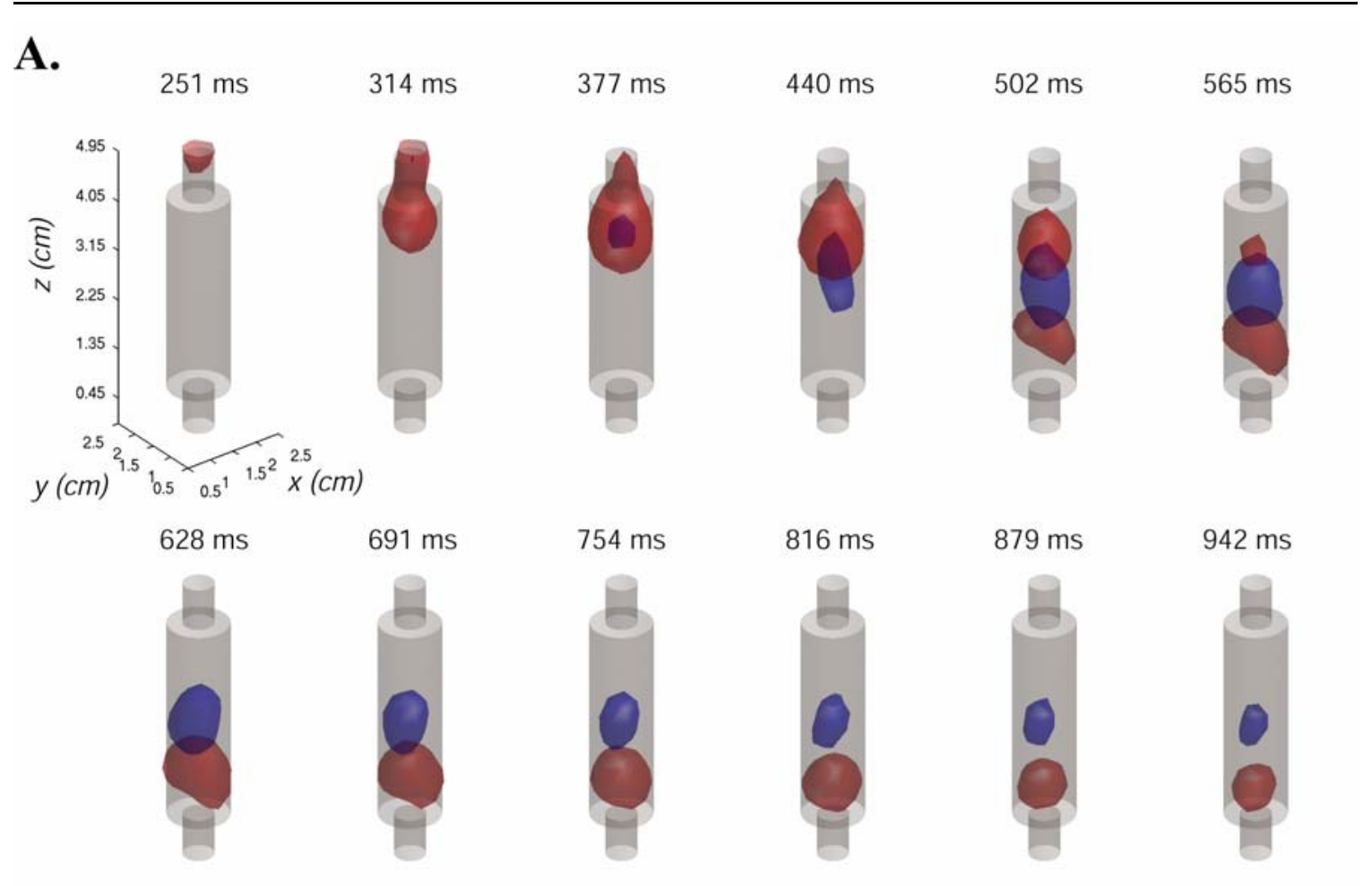

B.
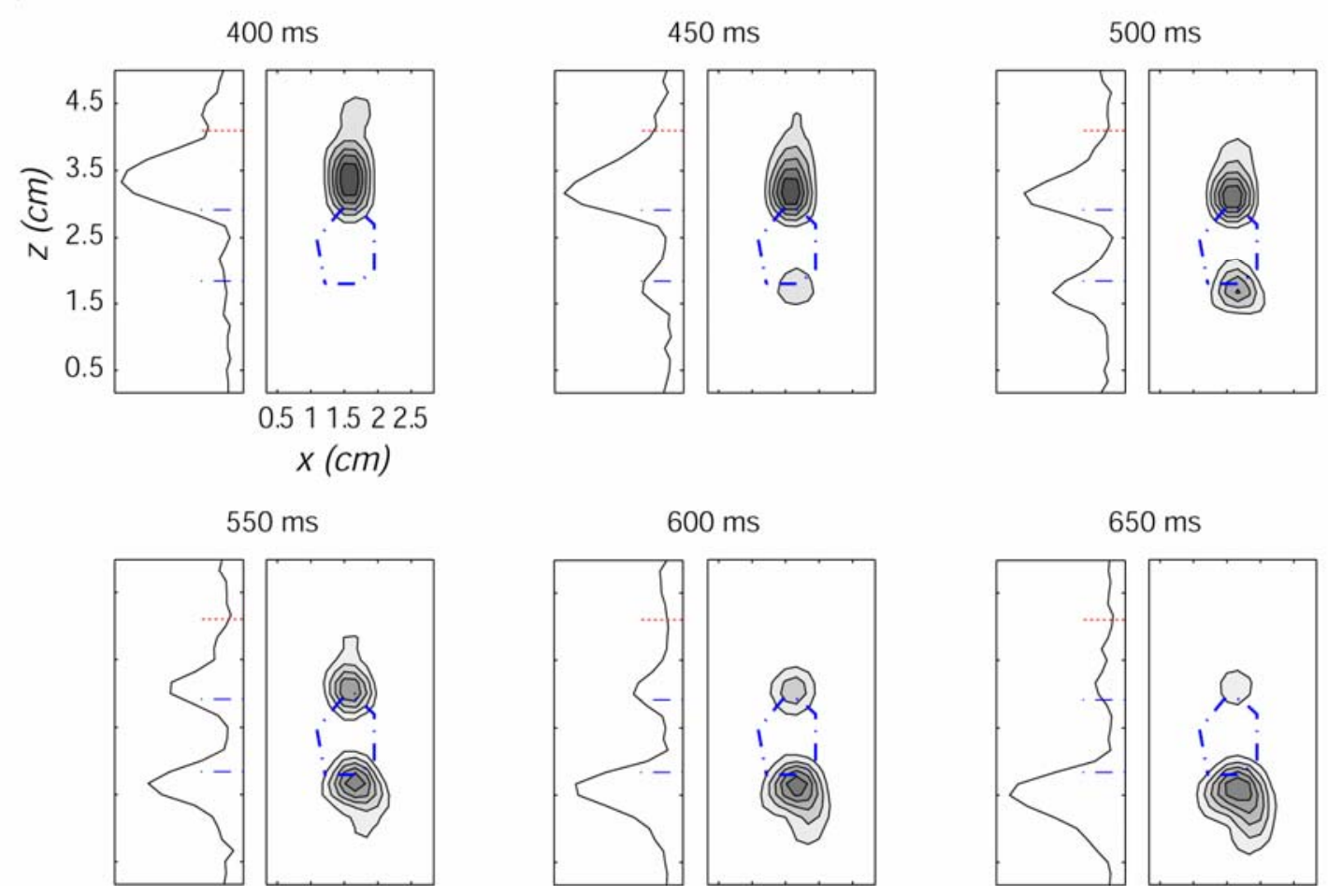

\section{FIGURE 3}



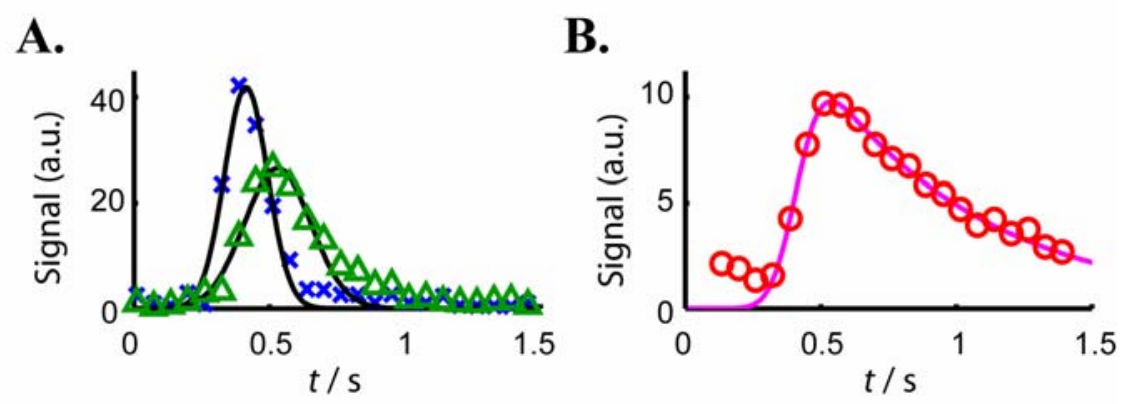

C.



E.

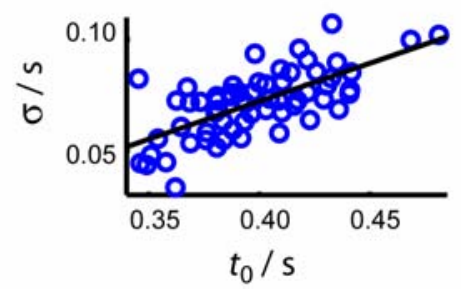

D.

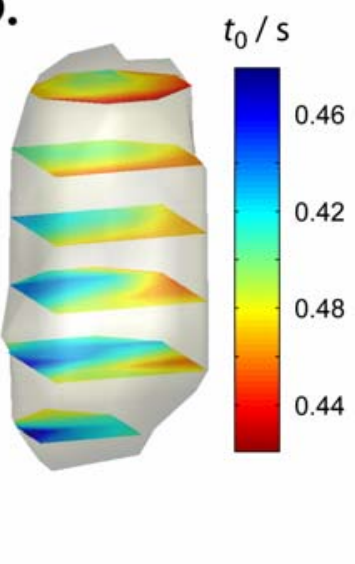

F.

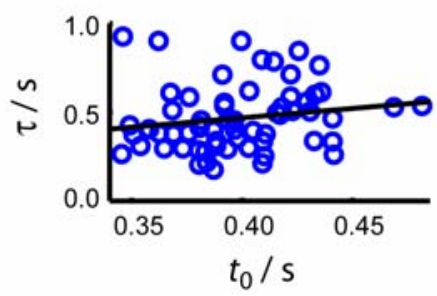

\section{FIGURE 4}

